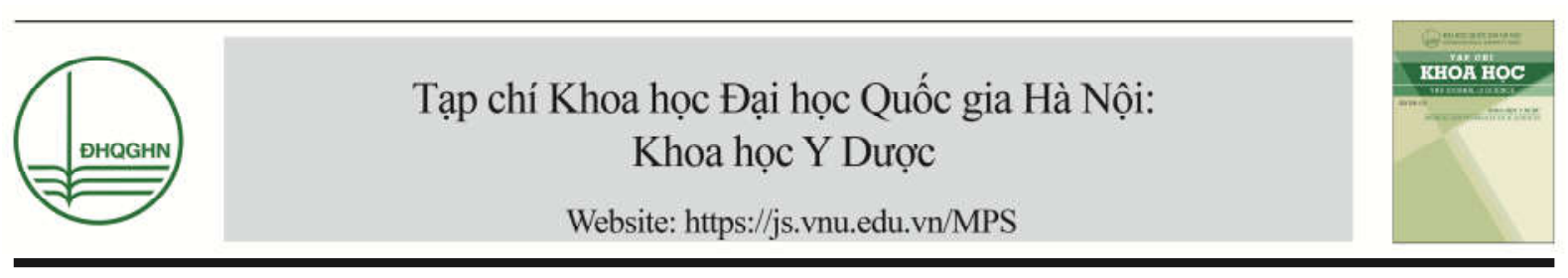

\title{
Một số hợp chất phân lập từ phân đoạn dịch chiết ethylacetat phần trên mặt đất của cây Chua me đất hoa vàng (Oxalis corniculata L.)
}

\author{
Vũ Đức Lợi ${ }^{1, *}$, Đặng Thị Quỳnh Nga ${ }^{1}$, Đỗ Thị Mai Hương ${ }^{2}$, Nguyễn Quốc Huy ${ }^{2}$ \\ ${ }^{1}$ Khoa Y Dược, Đại học Quốc gia Hà Nội, 144 Xuân Thủy, Cầu Giấy, Hà Nội, Việt Nam \\ ${ }^{2}$ Truò̀ng Đại học Dược Hà Nội, 13-15 Lê Thánh Tông, Hoàn Kiếm, Hà Nội, Việt Nam
}

Nhận ngày 14 tháng 4 năm 2018

Chỉnh sửa ngày 28 tháng 4 năm 2018; Chấp nhận đăng ngày 12 tháng 6 năm 2018

\begin{abstract}
Tóm tắt: Từ phân đoạn dịch chiết ethylacetat phần trên mặt đất của cây Chua me đất hoa vàng thu hái ở tỉnh Hà Tĩnh và bằng phương pháp sắc ký cột đã phân lập được 3 hợp chất. Cấu trúc hóa học của các hợp chất này được xác định bằng phương pháp phổ như: phổ khối, phổ cộng hưởng từ hạt nhân. Các chất được xác định là: acid eburicoic (1), 24- methylenecholest-4-en-3 $\beta, 6 \beta$-diol (2), $3 \beta$-hydroxylanosta-8,24-dien-21-oic acid (3). Các hợp chất này lần đầu tiên được phân lập từ phần trên mặt đất của cây Chua me đất hoa vàng.
\end{abstract}

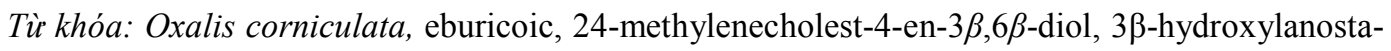
8,24-dien-21-oic.

\section{1. Đặt vấn đề}

Trên thế giới, chi Oxalis (họ Oxalidaceae) bao gồm hơn 900 loài, phân bố ở vùng nhiệt đới và cận nhiệt đới [1]. Hiện nay, ở Việt Nam phát hiện có 4 loài thuộc chi Oxalis, bao gồm: Chua me núi (Oxalis acetosella L.), Chua me đất hoa hồng (Oxalis corymbosa DC.), Chua me đất hoa vàng (Oxalis corniculata $\mathrm{L}$.) và Me đất đỏ (Oxalis deppei $\mathrm{Sw}$.), trong đó có 3 loài được dùng làm thuốc. Cây Chua me đất hoa vàng là loài phổ biến nhất [2]. Một số nghiên cứu cho thây cây Chua me đất có chứa nhóm chất như

\footnotetext{
* Tác giả liên hệ. ĐT.: 84-917879959.

Email: Ducloi82@gmail.com

https://doi.org/10.25073/2588-1132/vnumps.4112
}

flavonoid, tanin, ...[1, 3$]$ và có tác dụng chống oxy hóa, chống viêm [4]. Cho đến nay, các công trình nghiên cứu đã công bố về thành phần hóa học, tác dụng sinh học của cây Chua me đất hoa vàng ở Việt Nam cũng như trên thế giới còn khá ít. Bài báo này trình bày một số kết quả nghiên cứu về thành phần hóa học góp phần bổ sung thêm dữ liệu về cây chua me đất hoa vàng và hướng nghiên cứu tác dụng sinh học của cây này.

\section{Nguyên liệu và phương pháp nghiên cứu}

\subsection{Nguyên liệu}

Cây Chua me đất hoa vàng được thu hái vào tháng 7 năm 2016 tại xã Cẩm Sơn, huyện Cẩm 
Xuyên, tỉnh Hà Tĩnh, phần trên mặt đất của cây được phơi sấy khô, bảo quản trong túi nilon kín. Mẫu thực vật (số hiệu: Vũ Đức Lợi 11) đã được Viện Tài nguyên sinh vật, Viện Hàn lâm Khoa học và Công nghệ Việt Nam liệu giám định tên khoa học là: Oxalis corniculata L., họ Chua me đất (Oxalidaceae), mẫu cũng đang được lưu giữ tại Khoa Y Dược, ĐHQGHN.

\subsection{Hóa chất, thiết bị}

- Sắc ký lớp mỏng: sử dụng bản mỏng nhôm tráng sẵn silicagel $60 \mathrm{~F}_{254}$ Merck, độ dày $0,2 \mathrm{~mm}$. Sau khi triển khai sắc ký, bản mỏng được kiểm tra bằng đèn tử ngoại ở bước sóng $254,365 \mathrm{~nm}$ sau đó hiện màu bằng thuốc thử là dung dịch $\mathrm{H}_{2} \mathrm{SO}_{4} 10 \%$ trong ethanol.

- Sắc ký cột: sắc ký cột sử dụng silicagel cõ hạt $0.063-0.200 \mathrm{~mm}$ (Merck) và cỡ hạt 0,040 0,063 mm (Merck) với các loại cột sắc ký có kích cỡ khác nhau. Cột sephadex LH-20.

- Phổ cộng hưởng từ hạt nhân NMR được ghi trên máy Bruker Avance $500 \mathrm{MHz}$ tại Viện Hóa học, Viện Hàn lâm Khoa học và Công nghệ Việt Nam.

- Phổ khối ESI-MS đo trên máy Varian Agilent 1100 LC-MSD tại Viện Hóa học, Viện Hàn lâm Khoa học và Công nghệ Việt Nam.

- Đo nhiệt độ nóng chảy trên máy SMP10 BioCote tại Khoa Y Dược, ĐHQGHN.

\subsection{Phuoong pháp chiết xuất và phân lập}

Phần trên mặt đất của cây chua me đất được phơi sấy khô, nghiền thành bột $(2,4 \mathrm{~kg})$, ngâm chiết trong methanol $(8,0$ lít $\times 3$ lần). Dịch chiết methanol sau đó được quay cất loại dung môi dưới áp suất giảm thu được $196 \mathrm{~g}$ dịch cô. Lấy $176 \mathrm{~g}$ dịch cô này hoà vào 1,5 lít hỗn hợp $\mathrm{MeOH}: \mathrm{H}_{2} \mathrm{O}(1 / 1)$ rồi chiết phân bố lần lượt bằng các dung môi $n$-hexan, dichloromethan, ethyl acetat. Sau khi cất loại dung môi dưới áp suất giảm thu được các cặn chiết n-hexan (60 $\mathrm{g})$, dichlomethan (28 g), ethyl acetat (18 g) và dịch nước còn lại.

Từ $15,0 \mathrm{~g}$ cặn chiết ethyl acetat tiến hành sắc ký cột trên silicagel pha thường với hệ dung môi rửa giải aceton:chloroform: methanol
3/1/0,1; thu được sáu phân đoạn $\mathrm{A}(2,2 \mathrm{~g}), \mathrm{B}$ $(1,6 \mathrm{~g}), \mathrm{C}(800 \mathrm{mg}), \mathrm{D}(1,4 \mathrm{~g}), \mathrm{E}(1,2), \mathrm{F}(1,6$ g). Phân đoạn $\mathrm{A}$ phân tách trên sắc ký cột pha thường, hệ dung môi $\mathrm{CHCl}_{3}-\mathrm{MeOH}(20: 1)$ thu được 3 phân đoạn chính (A1, A2, A3). Phân đoạn $\mathrm{A} 1$ được phân tách qua cột sắc ký pha thường, dung môi $\mathrm{CHCl}_{3}-\mathrm{MeOH}(15: 1)$ thu được 4 phân đoạn nhỏ A1.1, A1.2, A1.3 và $\mathrm{A} 1.4$. Khi tách phân đoạn $\mathrm{A} 1.1$ bằng sắc ký cột pha đảo, hệ dung môi $\mathrm{MeOH}: \mathrm{H}_{2} \mathrm{O}(3: 2$, v/v) thu được hợp chất 1 (15 mg). Phân đoạn A1.2 được phân tách trên cột sắc ký pha thường, với hệ dung môi rửa giải $\mathrm{CH}_{2} \mathrm{Cl}_{2}$ : Aceton $(2: 1, \mathrm{v} / \mathrm{v})$ thu được hợp chất 2 (20mg). Phân đoạn A1.3 được phân tách bằng sắc ký cột pha đảo, với hệ dung môi rửa giải $\mathrm{ACN}: \mathrm{H}_{2} \mathrm{O}(90: 10, \mathrm{v} / \mathrm{v})$ thu được hợp chất 3 (12mg).

\section{Kết quả và thảo luận}

\section{Hợp chất 1:}

Tinh thể màu trắng; $\mathrm{t}_{\mathrm{nc}}=280-283{ }^{\circ} \mathrm{C}$;

IR $(\mathrm{KBr}) v_{\max }\left(\mathrm{cm}^{-1}\right): 3332,1720,1648$, 890; ESI-MS $m / z 469$ [M] ${ }^{+}$

${ }^{1} \mathrm{H}-\mathrm{NMR}\left(400 \mathrm{MHz}\right.$, pyridine- $\left.d_{5}\right)(\delta \mathrm{ppm})$ : 4,88 $(2 \mathrm{H}, b r s, \mathrm{H}-31) ; 3,41(1 \mathrm{H}, t, J=10,5 \mathrm{~Hz}$, H-3); 2,62 (1H, td, $J=10,5$; 7,0 Hz, H-20); 2,49$2,39(1 \mathrm{H}, m, \mathrm{H}-17) ; 2,33-2,23$ (2H, $m, \mathrm{H}-23$, 25); 2,09-2,04 (1H, $m, \mathrm{H}-7 \beta, 16,22) ; 2,01-1,91$ $(2 \mathrm{H}, m, \mathrm{H}-8,11,12) ; 1,81(2 \mathrm{H}, d t, J=7,5,3,5$ $\mathrm{Hz}, \mathrm{H}-2) ; 1,67$ (1H, $m, \mathrm{H}-6 \alpha, 15 \alpha) ; 1,65$ (3H, $s$, $\mathrm{H}-27) ; 1,62-1,44(1 \mathrm{H}, m, \mathrm{H}-6 \beta, 7 \alpha) ; 1,60(3 \mathrm{H}$, d, $J=7 \mathrm{~Hz}, \mathrm{H}-26) ; 1,27(1 \mathrm{H}, t d, J=12,5 \mathrm{~Hz}, \mathrm{H}-$ $15 \beta) ; 1,23$ (3H, $s, \mathrm{H}-30)$; 1,18 (1H, $s, \mathrm{H}-1 \alpha)$; $1,14(1 \mathrm{H}, d d, J=11 \mathrm{~Hz}, \mathrm{H}-5) ; 1,06(3 \mathrm{H}, s, \mathrm{H}-$ 18); 1,00 (3H, $s, \mathrm{H}-19)$;

${ }^{13} \mathrm{C}-\mathrm{NMR}\left(100 \mathrm{MHz}\right.$, pyridine- $\left.d_{5}\right)(\delta \mathrm{ppm})$ : 191,3 (C-21); 155,9 (C-24); 135,4 (C-8), 134,3 (C-9); 107,0 (C-31); 78,0 (C-3); 50,9 (C-5); 49,8 (C-14); 49,2 (C-20); 47,7 (C-17); 44,9 (C13); 39,5 (C-4); 37,4 (C-10); 36,1 (C-1); 34,2 (C-25); 31,8 (C-22); 30,9 (C-15); 29,3 (C-12); 28,7 (C-2); 28,6 (C-29); 27,5 (C-16); 26,8 (C7); 24,5 (C-30); 22,0 (C-26); 21,9 (C-27); 21,3 (C-11); 19,4 (C-19); 18,7 (C-6); 16,4 (C-18). 
Hợp chất 2:

Chất bột vô định hình, màu trắng. $[\alpha]^{25}{ }_{\mathrm{D}}=-$ $21,0\left(\mathrm{c}=0,1, \mathrm{CHCl}_{3}\right)$

Công thức phân tử: $\mathrm{C}_{28} \mathrm{H}_{46} \mathrm{O}_{2}$. Khối lượng phân tử: 414

${ }^{13} \mathrm{C}-\mathrm{NMR}\left(125 \mathrm{MHz}, \mathrm{CDCl}_{3}\right)(\delta \mathrm{ppm})$ : $39,7(\mathrm{C}-1) ; \quad 30,1(\mathrm{C}-2) ; \quad 73,7(\mathrm{C}-3) ; 129,9(\mathrm{C}-4)$; 147,1(C-5); 67,5(C-6); 37,7(C-7); 28,4(C-8); 55,0(C-9); 37,3(C-10); 21,3(C-11); 40,7(C-12); $42,8(\mathrm{C}-13) ; \quad 56,4(\mathrm{C}-14) ; \quad 24,5(\mathrm{C}-15) ; \quad 28,5(\mathrm{C}-$ $16) ; \quad 56,3(\mathrm{C}-17) ; \quad 12,2(\mathrm{C}-18) ; \quad 21,6(\mathrm{C}-19)$; $36,0(\mathrm{C}-20) ; 18,9(\mathrm{C}-21) ; 35,0(\mathrm{C}-22) ; 30,1(\mathrm{C}-$
$23) ; \quad 156,7(\mathrm{C}-24) ; \quad 34,0(\mathrm{C}-25) ; \quad 22,6(\mathrm{C}-26)$; 22,1(C-27); 106,6(C-28).

${ }^{1} \mathrm{H}-\mathrm{NMR}\left(500 \mathrm{MHz}, \mathrm{CDCl}_{3}\right)(\delta \mathrm{ppm})$ : 1,16(1H, m, H-1); 1,97(1H, m, H-2); 5,99(1H, s, H-4); 1,38(1H, m, H-7); 1,52(1H, m, H-8); $0,87(1 \mathrm{H}, \mathrm{m}, \mathrm{H}-9) ; 1,49(1 \mathrm{H}, \mathrm{m}, \mathrm{H}-11) ; 1,28$ $(1 \mathrm{H}, \mathrm{m}, \mathrm{H}-12) ; 1,13(1 \mathrm{H}, \mathrm{m}, \mathrm{H}-15) ; 1,23(1 \mathrm{H}$, m, H-16); 0,72(1H, s, H-18); 1,54(1H, s, H-19); $1,41(1 \mathrm{H}, \mathrm{m}, \mathrm{H}-20) ; 0,99(1 \mathrm{H}, \mathrm{d}, \mathrm{J}=6,5 \mathrm{~Hz}, \mathrm{H}-$ $21) ; 1,22(1 \mathrm{H}, \mathrm{m}, \mathrm{H}-22) ; 1,97(1 \mathrm{H}, \mathrm{m}, \mathrm{H}-23)$; $2,28(1 \mathrm{H}, \mathrm{m}, \mathrm{H}-25) ; 0,88(1 \mathrm{H}, \mathrm{d}, \mathrm{J}=6,5 \mathrm{~Hz}, \mathrm{H}-$ 26); $0,89(1 \mathrm{H}, \mathrm{d}, \mathrm{J}=6,5 \mathrm{~Hz}, \mathrm{H}-27) ; 4,84(1 \mathrm{H}, \mathrm{d}$, $\mathrm{J}=8,0 \mathrm{~Hz}, \mathrm{H}-28$ ).

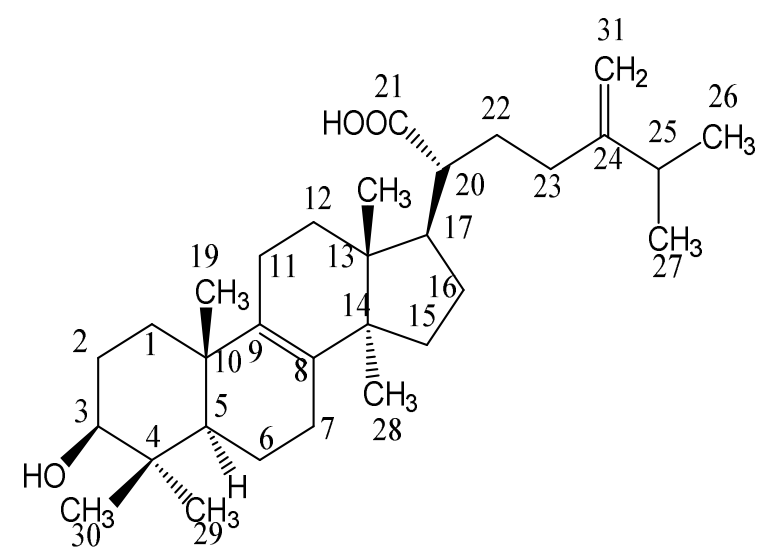<smiles>C=C(CC[C@@H](C)[C@H]1CCC2C3C[C@H](O)C4=C[C@@H](O)CC[C@]4(C)C3C=C[C@@]21C)C(C)C</smiles>

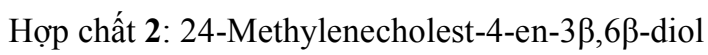<smiles>CC[C@]12C3=C(C=C[C@]4(C)[C@@H]([C@H](CCC=C(C)C)C(=O)O)CC[C@]34C)[C@@]1(C)CC[C@@H](O)C2(C)C</smiles>

Hợp chất 3: Acid 3ß-hydroxylanosta-8,24-dien-21-oic

Hình 1. Cấu trúc của các hợp chất 1-3. 
Hợp chất 3:

Tinh thể màu trắng; $\mathrm{t}_{\mathrm{nc}}=258-260^{\circ} \mathrm{C}$;

IR $(\mathrm{KBr}) v_{\max }\left(\mathrm{cm}^{-1}\right): 3200(-\mathrm{OH}), 1718$, 1632; ESI-MS $m / z 455[\mathrm{M}]^{+}$;

${ }^{1} \mathrm{H}-\mathrm{NMR}\left(400 \mathrm{MHz}\right.$, pyridine- $\left.d_{5}\right)(\delta \mathrm{ppm})$ : $5,32(1 \mathrm{H}, t, \mathrm{H}-24) ; 3,40(1 \mathrm{H}, t, J=9,5 \mathrm{~Hz}, \mathrm{H}-3)$; $2,61(1 \mathrm{H}, t d, J=9,5 ; 4,0 \mathrm{~Hz}, \mathrm{H}-20) ; 2,46-2,35$ $(2 \mathrm{H}, m, \mathrm{H}-16,17,23 \mathrm{a}) ; 2,29-2,25(1 \mathrm{H}, m, \mathrm{H}-$ $23 \mathrm{~b}) ; 2,06(2 \mathrm{H}, t, J=5,5 ; 3,5 \mathrm{~Hz}, \mathrm{H}-7)$; $1,97-$ $1,92(2 \mathrm{H}, m, \mathrm{H}-11,12,22 \mathrm{a}) ; 1,85-1,44(1 \mathrm{H}, m$, $\mathrm{H}-1 \mathrm{~b}) ; 1,81(2 \mathrm{H}, d t, J=9,5 ; 4,0 \mathrm{~Hz}, \mathrm{H}-2) ; 1,80$ $(1 \mathrm{H}, d t, J=9,5 ; 4,0 \mathrm{~Hz}, \mathrm{H}-1 \mathrm{a}) ; 1,78-1,68(1 \mathrm{H}, m$, $\mathrm{H}-6,15 \mathrm{a}, 22 \mathrm{~b}) ; 1,65$ (3H, $s, \mathrm{H}-27) ; 1,60$ (3H, $s$, $\mathrm{H}-26) ; 1,26(1 \mathrm{H}, t d, \mathrm{H}-15) ; 1,23(3 \mathrm{H}, s, \mathrm{H}-30)$; $1,15(1 \mathrm{H}, t, \mathrm{H}-5) ; 1,06(3 \mathrm{H}, s, \mathrm{H}-18,28) ; 1,00$ (3H, $s, \mathrm{H}-19) ; 0,99$ (3H, $s, \mathrm{H}-29)$.

${ }^{13} \mathrm{C}-\mathrm{NMR}\left(100 \mathrm{MHz}\right.$, pyridine- $\left.d_{5}\right)(\delta \mathrm{ppm})$ : 178,7 (C-21); 135,0 (C-8); 134,3 (C-9); 131,7 (C-25); 124,9 (C-24); 77,8 (C-3); 50,9 (C-5); 49,8 (C-14); 49, 1 (C-20); 47,7 (C-17); 44,9 (C13); 39,5 (C-4); 37,4 (C-10); 36,1 (C-1); 33,3 (C-22); 30,9 (C-15); 29,4 (C-12); 28,7 (C-2); 28,6 (C-28); 27,5 (C-16); 26,8 (C-7); 26,7 (C23); 25,8 (C-26); 24,5 (C-30); 21,2 (C-11); 19,4 (C-19); 18,7 (C-6); 17,7 (C-27) 16,4 (C-29); 16,4 (C-18).

Hợp chất 1: Acid eburicoic

Là tinh thể không màu, nhiệt độ nóng chảy 280-283 ${ }^{\circ} \mathrm{C}$. Phổ khối lượng ESI-MS của hợp chất 1 cho pic ion $m / z 469[\mathrm{M}]^{+}$tương ứng với công thức phân tử $\mathrm{C}_{31} \mathrm{H}_{50} \mathrm{O}_{3}$. Phổ hồng ngoại của hợp chất 1 có dải hấp thụ đặc trưng của nhóm exometylen $\left(=\mathrm{CH}_{2}\right)$ tại $890 \mathrm{~cm}^{-1}$; của nhóm cacbonyl $(\mathrm{C}=\mathrm{O})$ tại $1720 \mathrm{~cm}^{-1}$ và của nhóm hydroxyl (-OH) ở $3332 \mathrm{~cm}^{-1}$. Phổ ${ }^{1} \mathrm{H}-$ NMR của hợp chất 1 cho thấy tín hiệu của 5 nhóm metyl ở độ dịch chuyển $\delta_{\mathrm{H}} 1,06$ ppm $(\mathrm{H}-$ 28), 1,00ppm (H-29), 1,23 ppm (H-30), 1,60 ppm (H-26), 1,65 ppm (H-27) và hai proton exometylen tại $\delta_{\mathrm{H}} 4,88$ ppm và $4,92 \mathrm{ppm}(\mathrm{H}-$ 31). Ngoài ra, phổ ${ }^{1} \mathrm{H}-\mathrm{NMR}$ còn xuất hiện tín hiệu của proton carbinol tại $\delta_{\mathrm{H}} 3,45(1 \mathrm{H}, \mathrm{t}, \mathrm{H}-$ 3). Phổ ${ }^{13} \mathrm{C}-\mathrm{NMR}$, DEPT của hợp chất $\mathbf{1}$ xuất hiện tín hiệu của 31 carbon, bao gồm 5 carbon metin, 10 carbon metilen, 7 carbon metyl, 4 carbon olefinic, 1 carbonyl và 4 carbon bậc 4 . Tín hiệu phổ ${ }^{13} \mathrm{C}-\mathrm{NMR}$ của hợp chất $\mathbf{1}$ cho thấy tín hiệu của 5 carbon nhóm metyl tại C-18 $\left(\delta_{\mathrm{C}}\right.$ $16,4 \mathrm{ppm}), \mathrm{C}-19\left(\delta_{\mathrm{C}} 19,4 \mathrm{ppm}\right), \mathrm{C}-28\left(\delta_{\mathrm{C}} 28,6\right.$ ppm), C-29 ( $\left.\delta_{\mathrm{C}} 16,4 \mathrm{ppm}\right), \mathrm{C}-30\left(\delta_{\mathrm{C}} 24,5 \mathrm{ppm}\right)$, đồng thời các tín hiệu cacbon còn lại cho thấy cấu trúc gồm một hệ thống bốn vòng trong đó có một liên kết đôi tại vị trí C-8 $\left(\delta_{\mathrm{C}} 135,4 \mathrm{ppm}\right)$ và $\mathrm{C}-9\left(\delta_{\mathrm{C}} 134,3 \mathrm{ppm}\right)$, một liên kết đôi tại $\mathrm{C}$ $31\left(\delta_{\mathrm{C}} 107,0 \mathrm{ppm}\right)$ và $\mathrm{C}-24\left(\delta_{\mathrm{C}} 155,9 \mathrm{ppm}\right)$ và một cacbonyl tại C-21 ( $\delta_{\mathrm{C}} 191,3$ ppm). Phổ ${ }^{13} \mathrm{C}$ NMR còn xuất hiện tín hiệu tại $\delta_{\mathrm{C}} 78,0 \mathrm{ppm}$ là tín hiệu của $\mathrm{C}-3$ có gắn nhóm hydroxyl. Ngoài ra, trên phổ $\mathrm{HMBC}$ cho thấy sự tương tác giữa các proton và carbon trong phân tử của hợp chất 1. Kết hợp phổ ${ }^{1} \mathrm{H}-\mathrm{NMR},{ }^{13} \mathrm{C}-\mathrm{NMR}$ và $\mathrm{DEPT}$, HSQC, HMBC cho thấy hợp chất $\mathbf{1}$ là một dẫn xuất của lanosterol. Từ số liệu phổ như trên và so sánh với tài liệu [5], có thể xác định cấu trúc của hợp chất 1 là: acid eburicoic.

Hợp chất 2: 24-Methylenecholest-4-en$3 \beta, 6 \beta$-diol

Hợp chất 2 thu được dưới dạng bột màu trắng. Phổ ${ }^{1} \mathrm{H}-\mathrm{NMR}$ của hợp chất $\mathbf{2}$ cho biết sự có mặt của 5 nhóm methyl tại $\delta_{\mathrm{H}} 0,72(3 \mathrm{H}, \mathrm{s}, \mathrm{H}-$ 18), 1,54 ( $3 \mathrm{H}, \mathrm{s}, \mathrm{H}-19), 0,99(3 \mathrm{H}, \mathrm{d}, J=6,5 \mathrm{~Hz}$, $\mathrm{H}-21), 0,88(3 \mathrm{H}, \mathrm{d}, J=6,5 \mathrm{~Hz}, \mathrm{H}-26)$ và 0,89 $(3 \mathrm{H}, \mathrm{d}, J=6,5 \mathrm{~Hz}, \mathrm{H}-27) ; 1$ proton olefin tại $\delta_{\mathrm{H}}$ $5,99(1 \mathrm{H}, \mathrm{s}, \mathrm{H}-4)$; và 2 proton oxymethine tại $\delta_{\mathrm{H}}$ 4,55 $(2 \mathrm{H}, \mathrm{m})$. Phổ ${ }^{13} \mathrm{C}-\mathrm{NMR}$ của hợp chất 2 cho thấy sự có mặt của 28 carbon bao gồm 5 carbon methyl, 10 methylene, 9 methine và 4 carbon bậc 4 . Tương tác $\mathrm{HMBC}$ giữa $\mathrm{H}-26\left(\delta_{\mathrm{H}} 0,88\right)$ và $\mathrm{H}-27\left(\delta_{\mathrm{H}} 0,89\right)$ và $\mathrm{C}-24\left(\delta_{\mathrm{C}} 156,72\right) / \mathrm{C}-25\left(\delta_{\mathrm{C}}\right.$ $34,08)$; giữa $\mathrm{H}-28 \quad\left(\delta_{\mathrm{H}} 4,84\right)$ và $\mathrm{C}-23 \quad\left(\delta_{\mathrm{C}}\right.$ $30,19) / \mathrm{C}-24\left(\delta_{\mathrm{C}} 156,72\right) / \mathrm{C}-25\left(\delta_{\mathrm{C}} 34,08\right)$ gợ ý sự có mặt của liên kết đôi tại $\mathrm{C}-24 / \mathrm{C}-28$ và hai nhóm methyl tại $\mathrm{C}-25$. Tương tác $\mathrm{HMBC}$ giữa $\mathrm{H}-19\left(\delta_{\mathrm{H}} 1,54\right)$ với $\mathrm{C}-1 \quad\left(\delta_{\mathrm{C}} 39,73\right) / \mathrm{C}-5 \quad\left(\delta_{\mathrm{C}}\right.$ $147,15) / \mathrm{C}-9\left(\delta_{\mathrm{C}} 55,03\right) / \mathrm{C}-10\left(\delta_{\mathrm{C}} 37,33\right)$; giữa $\mathrm{H}-3$ và $\mathrm{H}-6\left(\delta_{\mathrm{H}} 4,55\right)$ đến $\mathrm{C}-4\left(\delta_{\mathrm{C}} 129,97\right) / \mathrm{C}-5$ $\left(\delta_{\mathrm{C}} 147,15\right) / \mathrm{C}-7\left(\delta_{\mathrm{C}} 30,95\right) / \mathrm{C}-10\left(\delta_{\mathrm{C}} 37,39\right)$ cho phép xác định vị trí của 2 nhóm hydroxyl tại $\mathrm{C}$ 3 và $C-6$. Từ các phân tích phổ nêu trên kết hợp so sánh với hợp chất 24- methylenecholest-4en-3 $\beta, 6 \beta$-diol [6], cho thấy sự giống nhau về số 
liệu ${ }^{13} \mathrm{C}-\mathrm{NMR}$ ở các vị trí tương ứng. Vì vậy, cấu trúc của hợp chất 2 được xác định là 24methylenecholest-4-en-3 $\beta, 6 \beta$-diol.

Hợp chất 3: Acid 3ß-hydroxylanosta-8,24dien-21-oic

Là chất bột màu trắng, điểm nóng chảy tại 258-260 ${ }^{\circ} \mathrm{C}$. Phổ khối lượng EI-MS của hợp chất 3 cho pic ion $m / z 455[\mathrm{M}]^{+}$tương ứng với công thức phân tử $\mathrm{C}_{30} \mathrm{H}_{48} \mathrm{O}_{3}$. Phổ ${ }^{1} \mathrm{H}-\mathrm{NMR}$ của hợp chất 3 có sự xuất hiện của các tín hiệu proton metyl tại 1,06 (3H, s, H-18), 1,00 (3H, s, $\mathrm{H}-19), 1,60(\mathrm{H}-26), 1,65(\mathrm{H}-27), 1,06(3 \mathrm{H}, \mathrm{s}$, H-28), 0,99 (3H, s, H-29), 1,23 (3H, s, H-30). Phổ ${ }^{13} \mathrm{C}$-NMR, DEPT của hợp chất 3 cho thấy tín hiệu của 30 carbon trong đó 4 carbon metin, 10 carbon metilen, 7 carbon metyl, 4 carbon olefinic, 1 carbonyl và 4 carbon bậc 4 . Các tin hiệu phổ cho thấy cấu trúc của hợp chất 3 cũng bao gồm một hệ thống bốn vòng, trong đó có một liên kết đôi tại vị trí C-8 $\left(\delta_{\mathrm{C}} 135,0 \mathrm{ppm}\right)$ và C-9 $\left(\delta_{C} 134,3\right.$ ppm). Hợp chất 3 có sự xuất hiện tín hiệu của hai carbon olefinic tại C-24 $\left(\delta_{\mathrm{C}}\right.$ $124,9 \mathrm{ppm})$ và $\mathrm{C}-25\left(\delta_{\mathrm{C}} 134,6 \mathrm{ppm}\right)$ và một carbonyl tại $\mathrm{C}-21\left(\delta_{\mathrm{C}} 178,7 \mathrm{ppm}\right)$. Ngoài ra, trên phổ HMBC cho thấy sự tương tác giữa các proton và carbon trong phân tử 3 . Kết hợp phổ ${ }^{1} \mathrm{H}-\mathrm{NMR},{ }^{13} \mathrm{C}-\mathrm{NMR}$ và $\mathrm{DEPT}, \mathrm{HSQC}, \mathrm{HMBC}$ cho thấy hợp chất $\mathbf{3}$ là một dẫn xuất của lanosterol. Từ số liệu phổ và so sánh với tài liệu [7], có thể xác định cấu trúc của hợp chất 3 phù hợp với cấu trúc của acid $3 \beta$-hydroxylanosta8,24-dien-21-oic.

\section{Kết luận}

Đã sử dụng phương pháp ngâm chiết với dung môi $\mathrm{MeOH}$ và phương pháp sắc ký cột phân lập được 3 hợp chất từ phần trên mặt đất của cây Chua me đất hoa vàng thu hái ở tỉnh Hà Tĩnh. Xác định cấu trúc các hợp chất phân lập được thông qua kết quả đo nhiệt độ nóng chảy, phổ khối, phổ cộng hưởng hạt nhân và so sánh với các dữ liệu công bố của các hợp chất liên quan. Ba hợp chất phân lập được xác định cấu trúc là: Acid eburicoic (1), 24methylenecholest-4-en-3 $\beta, 6 \beta$-diol (2), acid $3 \beta$ hydroxylanosta-8,24-dien-21-oic (3). Cả 3 hợp chất này đều lần đầu tiên phân lập được từ phần trên mặt đất của cây Chua me đất hoa vàng.

\section{Tài liệu tham khảo}

[1] Iqbal Hussain, Muhammad Imran, Nusrat Hussain, Amjad Hussain, Tooba Mahboob (2013), "Corniculatin A, a new flavonoidal glucoside from Oxalis corniculata", Revista Brasileira de Farmacognosia, 23(4), pp: 630-634.

[2] Đỗ Tất Lợi (2005), Những cây thuốc và vị thuốc Việt Nam, Nxb. Y học, Hà Nội, pp. 236-237.

[3] Merugu Srikanth, Tadigotla Swetha, Veeresh B (2012), "Phytochemistry and pharmacology of Oxalis corniculata Linn: A review", International journal of pharmaceutical sciences and research, 3(11), pp: 4077-4085.

[4] Muhammad Rashid Khan, Hina Zehra (2013), "Amelioration of $\mathrm{CCl}_{4}$-induced nephrotoxicity by Oxalis corniculata in rat", Experimental and Toxicologic Pathology, 65(3), pp: 327-334.

[5] Kirti Sheth, Philip Catalfomo, Leo A. Sciuchetti (1967), "Isolation and identification of eburicoic acid from Fomes pinicola", Journal of Pharmaceutical Sciences 56(12), pp.1656-1658.

[6] Faheem Amir, Yen Chin Koay and Wan Sinn Yam (2012), "Chemical Constituents and Biological Properties of the Marine Soft Coral Nephthea", Tropical Journal of Pharmaceutical Research, 11 (3): 499-517

[7] Rebamang A. Mosa, Adebola O. Oyedeji, Francis O. Shode, Mogie Singh and Andy R. Opoku (2011), "Triterpenes from the stem bark of Protorhus longifolia exhibit anti-platelet aggregation activity", African Journal of Pharmacy and Pharmacology, 5(24), pp. 2698-2714. 


\title{
Compounds Isolated from the Ethyl Acetate Fraction of the Aerial Parts of Oxalis corniculata L.
}

\author{
Vu Duc Loi ${ }^{1}$, Dang Thi Quynh Nga ${ }^{1}$, Do Thi Mai Huong ${ }^{2}$, Nguyen Quoc Huy ${ }^{2}$ \\ ${ }^{I}$ VNU School of Medicine and Pharmacy, 144 Xuan Thuy, Cau Giay, Hanoi, Vietnam \\ ${ }^{2}$ Hanoi University of Pharmacy, 13-15 Le Thanh Tong, Hoan Kiem, Hanoi, Vietnam
}

\begin{abstract}
The ethyl acetate fraction of the aerial parts of Oxalis corniculata L. (collected in Ha Tinh province) isolated three compounds (1-3) by chromatographic methods. Their structures were elucidated by spectroscopic methods, including MS and NMR. These compounds were identified as: Eburicoic acid (1); 24-methylenecholest-4-ene-3 $\beta, 6 \beta$-diol (2); and 3 $\beta$-hydroxylanosta-8.24-dien-21oic acid (3). These compounds were, for the first time, isolated from the aerial parts of Oxalis corniculata $L$.

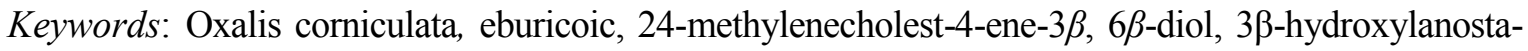
8,24-dien-21-oic.
\end{abstract}

